\title{
SINOPSIS DE LOS MELOIDAE (COLEOPTERA) DE CHIAPAS (MÉXICO) Y COMENTARIOS TAXONÓMICOS SOBRE EL GÉNERO DENIEROTA KASZAB, 1959
}

\author{
M. García-París ${ }^{1}$, J. L. Ruiz ${ }^{2}$, J. Vörös ${ }^{3} \&$ G. Parra-Olea ${ }^{4}$
}

\begin{abstract}
Resumen
La revisión de los ejemplares de la familia Meloidae de la pequeña colección entomológica del ECOSUR en San Cristóbal de las Casas (Chiapas, México), acompañada del examen de material chiapaneco del género Epicauta de la Colección Nacional de Insectos (CNIN-IBUNAM, Instituto de Biología, UNAM, México), material de Meloidae del Museo Húngaro de Historia Natural (HMNH, Magyar Természettudományi Múzeum, Budapest) y de la colección Marco A. Bologna (MAB, Università degli Studi Roma Tre, Italia), permite documentar por primera vez la presencia en el Estado de Chiapas de: Epicauta diana Pinto, 1991; Epicauta rufipennis (Chevrolat, 1834); Epicauta forticornis (Haag-Rutenberg, 1880); Epicauta distorta (Champion, 1892); Meloe tropicus Motschulsky, 1856; Denierota kraatzi (Haag-Rutenberg, 1880); Pyrota decorata (Haag-Rutenberg, 1880); Cissites auriculata (Champion, 1892) y Tetraonyx frontalis Chevrolat, 1833. De entre estas especies destacan las citas de M. tropicus que permiten confirmar la presencia de la especie en México y la de D. kraatzi que constituye el primer registro preciso del género Denierota en México. Por otra parte, la revisión del material utilizado por Z. Kaszab en la descripción del género Denierota (HNHM), acompañado del material de la colección M. Bologna y el examen de fotografías detalladas de los tipos de los dos taxones incluidos en el género Denierota, Lytta sanguineoguttata Haag-Rutenberg, 1880 y Lytta kraatzi Haag-Rutenberg, 1880, del Zoologische Staatssammlung de Münich (Alemania), nos permiten concluir que el género Denierota incluye una única especie descrita y nos permite formalizar la sinonimia de L. sanguineoguttata con L. kraatzi, resolviendo el largo y debatido problema taxonómico que suscitaba la identidad de $L$. kraatzi y el problema secundario de falta de localidad-tipo ("patria ignota") para esta especie.
\end{abstract}

Palabras-clave: Coleoptera, Meloidae, Denierota, Biodiversidad, Faunística, Taxonomía, América Central, México, Chiapas.

Museo Nacional de Ciencias Naturales. C.S.I.C., c/ José Gutiérrez Abascal, 2. 28006. Madrid. España. Instituto de Estudios Ceutíes. Paseo del Revellín, 30. Apartado 593. 51080 Ceuta. España.

Department of Zoology, Hungarian Natural History Museum. Baross u. 13. Budapest H-1088. Hungría

Instituto de Biología. Universidad Autónoma de México (UNAM). AP 70-153, CP 04510. Ciudad Universitaria. México D.F. México. 


\section{ABSTRACT \\ A synopsis of the Meloidae (Coleoptera) from Chiapas (México) and taxonomic comments on the genus Denierota Kaszab, 1959}

The revision of the specimens of the family Meloidae (Coleoptera) from the small Entomological Collection of ECOSUR at San Cristóbal de las Casas (Chiapas, Mexico), together with the revision of the material of Chiapas from the National Insect Collection (CNIN-IBUNAM, Instituto de Biología, UNAM, México) of the genus Epicauta, Hungarian Museum of Natural History (HMNH, Magyar Természettudományi Múzeum, Budapest) and Marco A. Bologna' collection (MAB, Università degli Studi Roma Tre, Italy), allow us to report the presence of Epicauta diana Pinto, 1991, Epicauta rufipennis (Chevrolat, 1834), Epicauta forticornis (Haag-Rutenberg, 1880), Epicauta distorta (Champion, 1892), Meloe tropicus Motschulsky, 1856, Denierota kraatzi (Haag-Rutenberg, 1880), Pyrota decorata (Haag-Rutenberg, 1880), Cissites auriculata (Champion, 1892) and Tetraonyx frontalis Chevrolat, 1833 for the first time in the State of Chiapas (México). Amongst these records, those of $M$. tropicus confirm the presence of the species in Mexico. The specimen of $D$. kraatzi represents the first precise record for the genus Denierota in México. The study of the material used by Z. Kaszab to describe the genus Denierota (HNHM), together with the specimens from M. Bologna's Collection and the study of photographs of the type specimens of Lytta sanguineoguttata Haag-Rutenberg, 1880 and Lytta kraatzi Haag-Rutenberg, 1880, the two taxa previously included in Denierota, from the Zoologische Staatssammlung of Münich (Germany), allow us to conclude that the genus includes only one described species, and to formalize the synonymy of L. sanguineoguttata with L. kraatzi, solving both the long standing problem of the identity of L. kraatzi and the secondary problem caused by the lack of type locality ("patria ignota") for the species.

Key words: Coleoptera, Meloidae, Denierota, Biodiversity, Faunistics, Central America, Mexico, Chiapas.

\section{Introducción}

Durante una corta visita al Estado de Chiapas (México) en agosto de 2007 se nos presentó la oportunidad de revisar la colección entomológica del ECOSUR (El Colegio de la Frontera Sur) en San Cristóbal de las Casas. El material de Meloidae de esta colección, que incluye algo menos de una treintena de ejemplares, está constituido por especímenes colectados a partir de 1970, procedentes sobre todo de áreas situadas alrededor de la zona central de los Altos de Chiapas. A pesar del escaso número de ejemplares estudiados, muchos de los registros que aportan corresponden a especies no citadas con anterioridad en el Estado de Chiapas: Epicauta forticornis (Haag-Rutenberg, 1880), Meloe tropicus Motschusky, 1856, Deniero-ta kraatzi (HaagRutenberg, 1880), Cissites auriculata (Champion, 1892) y Tetraonyx frontalis Chevrolat, 1833, lo que en algunos casos representa una considerable extensión de la distribución geográfica conocida de dichas especies (García-París et al., 2007). Entre estos registros destacan los de Meloe tropicus, que junto con los datos de la colección M. Bologna (Roma) suponen los primeros datos confirmados de la presencia de la especie en México. Además, el ejemplar de Denierota kraatzi supone la primera localidad concreta del género Denierota Kaszab, 1959 en México. La presencia del género Denierota en territorio mexicano fue señalada por Pinto (1984) y Pinto \& Bologna (1999) aunque sin precisar especies ni localidades.

A dicho material hemos creído conveniente añadir el material chiapaneco que hemos localizado en las colecciones del Museo Húngaro de Historia Natural (HMNH, Magyar Természettudományi Múzeum, Budapest), entre el que se incluye un ejemplar de Pyrota decorata (Haag-Rutenberg, 1880), que representa el primer registro de la especie en Chiapas. Además estos datos se complementan con los registros de ejemplares de Chiapas de los géneros Epicauta y Lytta que hemos estudiado de la Colección Nacional de Insectos del Instituto de Biología de la UNAM (México) (CNIN-IBUNAM), entre los que se incluyen los primeros registros chiapanecos de Epicauta (Epicauta) diana Pinto, 1991, Epicauta (Epicauta) rufipennis (Chevrolat, 1834) y Epicauta (Macrobasis) distorta (Champion, 1892). Adicionalmente, se incluyen datos de la Colección Marco A. Bologna (Università degli Studi Roma Tre, Italia) (Col. MAB), entre los que destacan los registros de $M$. tropicus ya comentados. 
Dada la complicada situación taxonómica de las especies incluidas en el género Denierota, aprovechamos la posibilidad de revisar el material utilizado por Z. Kaszab (HNHM) en la descripción original de este género, acompañado por los ejemplares de las colecciones de MAB y del ECOSUR, y fotografías de los tipos respectivos de $L$. sanguineoguttata Haag-Rutenberg, 1880 y L. kraatzi Haag-Rutenberg, 1880, del Zoologische Staatssammlung de Münich (Alemania), para revisar el estatus taxonómico de las mismas. Este estudio nos permite concluir que el género Denierota cuenta con una única especie descrita y formalizar por lo tanto la sinonimia de L. sanguineoguttata con L. kraatzi.

\section{Resultados y discusión}

Las especies y ejemplares examinados se enumeran a continuación, acompañadas de un breve comentario. Además del material que se comenta, se han examinado dos ejemplares de Epicauta (Epicauta) albolineata (Dugès, 1877) y uno de Epicauta (Epicauta) nigerrima (Dugès, 1870), colectados por C.C. Hoffmann en Chiapas, pero sin precisar localidad (Col. CNIN-IBUNAM). Ninguna de las dos especies está citada en el Estado de Chiapas aunque, al menos en el caso de E. albolineata, su presencia es muy probable. Consideramos que, mientras no existan otros registros, la presencia de estas dos especies en el Estado requiere confirmación y por lo tanto no tenemos en cuenta esos datos en los comentarios finales.

\section{Epicauta (Epicauta) major Pic, 1924}

México: Chiapas: Municipio Mal Paso: La Ceiva: 2-VI-1974, 1 ejemplar (A.R.R. leg.) [ECOSUR]. Mal Paso: La Selva, Km. 39: 1 ejemplar (C. A.R.R. leg.). [CNIN-IBUNAM]. Finca Prusia, Jaltenango: 10/12-V-1985, 3 ejemplares (F. Arias, H. Velasco, M. Vertiz leg.) [CNIN-IBUNAM]. La Canja, Chansayas: 19-VII1977, 1 ejemplar (P. Reyes leg.) / Epicauta major Pic, J. D. Pinto det., 1987/8 [CNIN-IBUNAM]. Municipio Tuzantán: Finca Irlanda, 1000-1200 msnm: 4-VI-74, 3 ejemplares, cafetal (J. Hendrichs S. leg.) [CNIN-IBUNAM]. Ocosingo: Km. 20 Ocosingo - Altamirano, 1077 msnm, 16 $48^{\prime} 45^{\prime \prime} \mathrm{N}-92^{\circ} 02^{\prime} 38^{\prime \prime} \mathrm{W}$ : 28-VI-2007, 2 ejemplares, bosque de pino-encino (C. Mayorga, G. Ortega, L. Cervantes leg.) [CNIN-IBUNAM]. Pichucalco: Km. 2 a Istapangajoya, $158 \mathrm{msnm}, 1^{\circ} 31^{\prime} 04^{\prime \prime N}-93^{\circ} 00^{\prime} 07^{\prime \prime} \mathrm{W}:$ 28-VI-2007, 1 ejemplar, vegetación secundaria (C. Mayorga, G. Ortega, L. Cervantes leg.) [CNIN-IBUNAM].

Especie muy variable de taxonomía complicada, que fue citada por varios autores bajo diversos nombres (Dugès, 1877, 1889; Champion, 1892; Denier, 1935b; Blackwelder, 1945), hasta que finalmente
Pinto (1991), en su revisión, definió su estatus taxonómico actual. La especie se extiende desde el noreste de México hasta Panamá, con registros en Costa Rica, Guatemala y Nicaragua (Pinto, 1991; Maes \& Huether, 2007). En México se ha citado en los estados de Campeche, Chiapas, Oaxaca, Quintana Roo, San Luis Potosí, Tabasco, Veracruz y Yucatán (Pinto, 1991; García-París et al., 2007). Las citas anteriores del Estado de Chiapas fueron publicadas por Pinto (1991). La actividad de los adultos se extiende desde mayo a julio.

\section{Epicauta (Epicauta) cupraeola (Dugès, 1869)}

México: Chiapas: Cascada de Agua, Km. 80 carretera Palenque - Bonampak (E. Barrera leg.) [CNIN-IBUNAM].

Especie de morfología muy variable y taxonomía complicada, que incluye en su sinonimia actual al menos cinco descripciones originales (Dugès, 1869a, 1870, 1877; Haag-Rutenberg, 1880; Horn, 1885).

La especie tiene una enorme distribución geográfica que se extiende desde Costa Rica hasta el extremo meridional de los Estados Unidos, incluyendo Nicaragua, Honduras, El Salvador, Guatemala y México (Pinto, 1991; Maes \& Huether, 2007). Su distribución en México es muy amplia y se ha registrado en casi todos los estados: Aguascalientes, Campeche, Chiapas, Chihuahua, Coahuila, Colima, Distrito Federal, Durango, Guanajuato, Guerrero, Jalisco, México, Michoacán, Morelos, Nayarit, Oaxaca, Puebla, Quintana Roo, San Luis Potosí, Sinaloa, Sonora, Veracruz, Yucatán y Zacatecas (Pinto, 1991; García-París et al., 2007). Las citas anteriores del Estado de Chiapas fueron publicadas por Pinto (1991).

\section{Epicauta (Epicauta) diana Pinto, 1991}

México: Chiapas: Berriozabal: 12/20-V-94, 1 ejemplar (C. Beutelspacher leg.) [CNIN-IBUNAM].

Especie endémica de México con muy pocos registros conocidos en Sinaloa y Oaxaca (Pinto, 1991). El ejemplar de Berriozabal de la colección CNIN-IBUNAM supone el primer registro para el estado de Chiapas y amplía su distribución potencial desde las zonas de la costa del Pacífico hacia el interior.

Epicauta (Epicauta) rufipennis (Chevrolat, 1834)

México: ChIAPAS: Tuxtla Gutiérrez - Rizo de Oro: 8-V-1994, 3 ejemplares (C.R. Beutelspacher leg.) [CNIN-IBUNAM].

Especie con poblaciones conocidas en México y Guatemala (Pinto, 1991). En México se ha citado 
en los estados de Chihuahua, Distrito Federal, Guanajuato, Guerrero, Hidalgo, Jalisco, México, Michoacán, Morelos, Nayarit, Oaxaca, Puebla, Sinaloa y Veracruzn (García-París et al., 2007). Los ejemplares estudiados de Chiapas presentan una coloración rojiza más patente que en otros individuos, aunque entran dentro de la variabilidad esperable de la especie. Estos ejemplares suponen el primer registro de E. rufipennis en Chiapas.

\section{Epicauta (Epicauta) hubbelli Werner, 1973}

México: ChiApas: Km. 84 carretera Tuxtla Gutiérrez - Villa Flores, 530 msnm: 12-VII-1988, 6 ejemplares (A. Cadena, L. Cervantes leg.) [CNIN-IBUNAM]. Rizo de Oro: 6/15-VI1994, 1 ejemplar (C.R. Beutelspacher) [CNIN-IBUNAM]. 3 km E Rizo de Oro: 3-VII-1981, 1 ejemplar (J.D. Pinto, Mathieu J., E. Fischer, J. La Salle) / Epicauta hubbelli Werner; J.D. Pinto det., 1987 [MAB].

Especie endémica de México, con poblaciones conocidas en Chiapas y Oaxaca (Pinto, 1991). Los ejemplares examinados se localizan dentro del área conocida para la distribución de la especie. La actividad de los adultos se concentra en junio y julio.

\section{Epicauta (Epicauta) teresa Mathieu, 1983}

MéXico: Chiapas: Km. 84 carretera Tuxtla Gutiérrez - Villa Flores, 530 msnm: 12-VII-1988, 6 ejemplares (A. Cadena, L. Cervantes leg.) [CNIN-IBUNAM]. El Sumidero: 17-VII-1984, 1 ejemplar (G. Gunther leg.) / Epicauta teresa Mathieu; J.D. Pinto det., 1987 [MAB].

Especie endémica de México, que como la especie anterior presenta poblaciones en Chiapas y Oaxaca (Pinto, 1991). Como en el caso anterior, los ejemplares examinados se localizan dentro del área conocida para la distribución de la especie.

Epicauta (Epicauta) vitticollis (Haag-Rutenberg, 1880)

MéXICo: ChIAPAs: Municipio Ocozocuautla: Reserva El Ocote: $2 \mathrm{~km}$ al SO de Cuauhtémoc: 7-X-1979, 1 ejemplar (O. Gómez N. leg.) [ECOSUR]. Reserva El Ocote: 30-V-1993, 1 ejemplar (E. Barrera leg.) [CNIN-IBUNAM]. Municipio Tuxtla Gutiérrez: Mal Paso: 2-VI-1974, 1 ejemplar (A.R.R. CIES leg.) [ECOSUR]. Mal Paso: La Selva: 1 al 2-VI-1974, 1 ejemplar (C.A.R.R.) [CNIN-IBUNAM]. Agua Azul: 1-V-1974, 2 ejemplares, col. nocturna (E. Barrera leg.) / Epicauta vitticollis Haag, J. D. Pinto det., 1987 [CNIN-IBUNAM]. Palenque: 22-VI-1979, 1 ejemplar (E. Barrera leg.) / Epicauta vitticollis Haag, J. D. Pinto det., 1987 [CNIN-IBUNAM]. Palenque, 600 m: 17/23-VI1994, 1 ejemplar (V. Maly leg.) (94/A/09, M-38) [MAB] [F]

Especie de amplia distribución en América central, citada en Belice, Guatemala, Honduras,
México y Nicaragua (Pinto, 1991; Maes \& Huether, 2007). En México se distribuye en los estados de Campeche, Chiapas, Oaxaca, Quintana Roo, Tabasco, Veracruz y Yucatán (García-París et al., 2007), donde puede causar daños a los cultivos de frijol (Sánchez Soto, 2001). Las citas anteriores en el Estado de Chiapas corresponden a Adams \& Selander (1979). La actividad de los adultos se extiende desde primeros de mayo hasta finales de junio, con un registro aislado en octubre.

\section{Epicauta (Macrobasis) candezi (Haag-Rutenberg, 1880)}

México: Chiapas: Municipio La Trinitaria: Lagunas de Montebello cerca de Cinco Lagos: 11-V-1994, 1 ejemplar (B. Gómez leg.) [ECOSUR]. Municipio Ocozocuautla: Reserva El Ocote: La Junta: 8-VI-1994, 1 ejemplar (O. Gómez N. leg.) [ECOSUR]. Municipio Ocozocuautla: Laguna Bélgica: 20-VI1996, red de golpeo, 1 ejemplar (O. Gómez N. leg.) [ECOSUR]. Municipio Ocozocuautla: R. Fracción El Chapapote: 20 km NO Ocozocuautla: 20-VI-1996, 2 ejemplares (O. Gómez N. leg.), 4 ejemplares (M. Girón leg.) [ECOSUR]. Municipio Tuxtla Gutiérrez: Agua Azul, camino al Sumidero: 29-V-1974, 1 ejemplar (Grupo CIES leg.) [ECOSUR].

Esta especie se incluyó en la sinonimia de Epicauta diversicornis (Haag-Rutenberg, 1880) hasta el trabajo de Werner (1949) donde se restituyó su estatus específico. Taxón conocido de El Salvador, Guatemala y México (Werner, 1949; Pinto, 1991) y posiblemente también presente en Costa Rica (Blackwelder, 1945). En México se ha citado por Werner (1949) y Pinto (1991) en los estados de Chiapas y Yucatán.

Todos los ejemplares examinados presentan el tegumento de la cabeza y tórax del mismo tono que el de los élitros. Según se desprende de los datos de captura del material estudiado, se trata de una especie frecuente en Chiapas a finales de primavera y principios del verano.

\section{Epicauta (Macrobasis) cinereiventris Champion, 1892}

México: Chiapas: Municipio Tuxtla Gutiérrez: Cerro Hueco: 14-X-1974, 1 ejemplar ("Grupo" leg.) [ECOSUR]. Cañón del Sumidero: 4-X-1974, 1 ejemplar ("Grupo" leg.) [ECOSUR]. Rancho del Sr. Coutiño en Terán: 5-XI-1974, 1 ejemplar (J.A. Domínguez leg.); 7-XI-1974, 1 ejemplar (“Grupo" leg.) [ECOSUR].

Epicauta cinereiventris está presente en México y Guatemala (Champion, 1892). Las citas mexicanas se distribuyen por los estados de Chiapas, Guerrero y Oaxaca (García-París et al., 2007). La cita publicada de Chiapas con anterioridad (Pinto, 1991) correspondía a un registro estatal, por lo que 
aquí se aportan los primeros registros concretos de la especie en Chiapas. La actividad de la especie en Chiapas se concentra en octubre y noviembre.

\section{Epicauta (Macrobasis) distorta (Champion, 1892)}

MéXICO: CHIAPAS: Escuintla: IV-1938, 3 ejemplares (P. Roveglia leg.) / Epicauta distorta Champion, F. Werner det. [CNIN-IBUNAM].

Especie ampliamente distribuida desde el sur de México hasta Costa Rica, con citas en Honduras y Nicaragua (Pinto, 1991; Maes \& Huether, 2007). En México únicamente había sido citada en los Estados de Guerrero y Veracruz (García-París et al., 2007), por lo que los ejemplares estudiados constituyen las primeras citas de la especie en Chiapas.

Epicauta (Macrobasis) forticornis (Haag-Rutenberg, 1880)

MéXICo: ChIAPAs: Municipio Tuxtla Gutiérrez: La Ceiva: 22IV-1974, 1 ejemplar (R. Álvarez leg.) [ECOSUR]. Jaltenango: 3-V-1940, 1 ejemplar [CNIN-IBUNAM].

Endemismo mexicano, que había sido citado hasta el momento en los estados de Guerrero, Morelos, Nayarit y Yucatán (Werner, 1949; Pinto, 1991). Los ejemplares estudiados representan los primeros registros para Chiapas.

Epicauta (Macrobasis) funesta (Chevrolat, 1834)

México: ChiApas: Municipio La Trinitaria: $30 \mathrm{~km}$ al SE de La Trinitaria: 29-IX-1994, ex Solanum, 1 ejemplar (R. Jones leg.) [ECOSUR]. Veracruz: Cascada Texolo: 5-XI-1976, 2 ejemplares, colecta diurna (Figueroa leg.) [Epicauta funesta (Chevr.), J. D. Pinto det., 1987] [CNIN-IBUNAM].

Especie endémica de México, citada con anterioridad en los estados de Chiapas, Puebla y Veracruz (Chevrolat, 1834; Dugès, 1869b, 1889; Champion, 1892; Selander \& Agafitei, 1982; Pinto, 1991). Las citas previas de Chiapas corresponden a una población localizada en "El Sumidero road, near Tuxtla Gutiérrez" (Selander \& Agafitei, 1982). El ejemplar examinado por nosotros de Chiapas, a diferencia de los ejemplares típicos del Estado de Veracruz (Colección CNIN-IBUNAM), presenta una serie de rasgos característicos: la mancha negra del tercio posterior de los élitros es de una extensión reducida y no toca el borde exterior del élitro y la coloración general de la pilosidad es pardoamarillenta en lugar del color grisáceo característico de la especie. Estos rasgos podrían entrar en el rango de variabilidad de E. funesta o representar una población diferenciada. En cualquier caso el examen de un único ejemplar no permite determinar este extremo por lo que hemos preferido mantener la identificación con reservas.

\section{Epicauta (Macrobasis) niveolineata (Haag-Rutenberg,} 1880)

MÉXICo: ChIAPAS: Cintalapa: 16-IX-1947, 1 ejemplar [CNINIBUNAM].

Esta especie presenta variabilidad marcada en la coloración del protorax, en la presencia de una banda apical clara en el ápice de los élitros y en la forma de las bandas longitudinales de los élitros, aunque no se ha determinado la existencia de un patrón geográfico claro. Se trata de una especie endémica de México (Pinto, 1991), extendida por los estados de Chiapas, Guerrero, Oaxaca y Veracruz. La cita publicada de Chiapas correspondía a un registro estatal (Pinto, 1991) por lo que se aporta el primer registro concreto de la especie.

\section{Meloe (Meloe) tropicus Motschulsky, 1856}

MéXico: Chiapas: Municipio San Cristóbal de Las Casas: San Antonio: Las Rosas: 13-XII-1995, bosque de pino-encino, 1 ejemplar (J. Godínez leg.) [ECOSUR]. San Cristóbal de las Casas: 28-VI-1985, 1 ejemplar (P. Brignoli leg.) / Meloe tropicus Motsch.; M.A. Bologna det. [MAB]. Chis. III-1938, 1 ejemplar (RF) (Dampf leg.) (7992-116) [MAB].

La distribución de esta especie se conocía desde Panamá hasta Guatemala, incluyendo registros en Costa Rica, El Salvador y Nicaragua (Pinto \& Selander, 1970). Champion (1891) incluyó en la serie tipo de Meloe dugesi Champion, 1891 dos ejemplares de $M$. tropicus procedentes al parecer de Xalapa (Veracruz), pero Pinto \& Selander (1970) decidieron no considerarlos como registros válidos, ya que según Bates (1884) muchos registros de dicha localidad provenían en realidad de otros lugares. Los ejemplares aquí estudiados permiten confirmar la presencia de la especie en México y constituyen los primeros registros del Estado de Chiapas.

Existe cierta variabilidad en algunos de los caracteres diagnósticos de la especie como por ejemplo la forma de la cabeza, que en el ejemplar de la colección del ECOSUR se aproxima a la de $M$. nebulosus, o en la anchura de los segmentos antenales de la hembra (Colección MAB). Sin embargo consideramos que el conjunto de caracteres responden a las características de la especie tal como consideran Pinto \& Selander (1970). 
Meloe (Treiodous) gracilicornis Champion, 1891

MéXico: Chiapas: San Cristóbal de las Casas: 2-X-1975, 1 ejemplar (R. Argano leg.) / Meloe (Treiodous) gracilicornis Champ.; M.A. Bologna det. 1995 [MAB]. San Cristóbal de las Casas: 13-VIII-1973, 1 ejemplar (R. Argano leg.) [MAB].

Especie conocida de México y Guatemala (Pinto \& Selander, 1970). En México se ha citado en los estados de Chiapas, Guerrero, Hidalgo, México, Nayarit, Oaxaca y Puebla (García-París et al., 2007). Las citas previas de Chiapas, que ya incluían la localidad aquí reseñada, fueron publicadas por Pinto \& Selander (1970).

\section{Meloe (Treiodous) laevis Leach, 1815}

México: Chiapas: San Cristóbal de las Casas: IX-1975, 1 ejemplar (V. Vomero leg.) [MAB].

Especie ampliamente distribuida desde Venezuela (Arnold, 1976) hasta el centro de los Estados Unidos, con citas en Panamá, Colombia, Costa Rica, Haití, Honduras, Guatemala, México, Nicaragua y República Dominicana (Pinto \& Selander, 1970; Maes \& Huether, 2007). En México se ha encontrado en casi todos los estados, incluidos: Aguascalientes, Chiapas, Chihuahua, Coahuila, Distrito Federal, Durango, Guanajuato, Guerrero, Hidalgo, Jalisco, México, Michoacán, Morelos, Nuevo León, Oaxaca, Puebla, Querétaro, San Luis Potosí, Sinaloa, Sonora, Veracruz y Zacatecas (García-París et al., 2007). Las citas previas del Estado de Chiapas corresponden a Pinto \& Selander (1970) y a Linsley et al. (1980).

\section{Denierota kraatzi (Haag-Rutenberg, 1880)}

México: Chiapas: Municipio Ángel Albino Corzo: Reserva El Triunfo, 1960 m: 10-VIII-1996, 1 ejemplar (A.R. Gillogly leg.) [ECOSUR]. MÉxICo (sin más precisión): 1 ejemplar (Pyrota kraatzi Haag; Z. Kaszab det.) [HNHM]. 1 ejemplar (Pyrota kraatzi ab. sanguineoguttata Haag; Z. Kaszab det.) [HNHM].

Guatemala: Chimaltenango: Acatenango: Quisache, 1750 m: 1-IX-1966, 1 ejemplar (T.W. Taylor leg.) / Denierota kraatzi (Haag-Rutenberg); M.A. Bologna det., 1995 [MAB]. Mpio. Acatenango: Quisache: V-1976, 1 ejemplar / Denierota sanguineoguttata (Haag-Rutenberg); H.R. Taylor det., 1976 [MAB]. SANTA Rosa: Santa Rosa: 1 ejemplar (Pyrota kraatzi Haag; Z. Kaszab det.) [HNHM].

Pinto (1984) y Pinto \& Bologna (1999) señalan la presencia del género Denierota Kaszab, 1959 en el sur de México, aunque sin indicar ni especie ni localidad precisa. Los datos que aquí se presentan constituyen por lo tanto las primeras citas concretas del género en México. El ejemplar examinado de
Chiapas presenta una coloración uniforme negroverdosa con reflejos verde metálicos.

Según Pinto \& Bologna (1999) el género Denierota, integrado actualmente en la tribu Pyrotini MacSwain, 1956 y distribuido por el sur de México, Guatemala y El Salvador, estaría representado por dos especies, que corresponderían a Denierota kraatzi (Haag-Rutenberg, 1880) y Denierota sanguineoguttata (Haag-Rutenberg, 1880). Estas dos especies fueron descritas originalmente dentro del género Lytta Fabricius, 1775. Sus respectivas descripciones originales son relativamente parecidas entre sí y el propio Haag-Rutenberg (1880: 40) comenta dicha similitud al final de la descripción de L. sanguineoguttata.

Casi simultáneamente, Kaszab (1959) y Selander (1960a) transfieren cada una de estas dos especies a sendos géneros nuevos. Así, Kaszab (1959) describe el género Denierota, donde sólo incluye a $L$. kraatzi, que designa como especie-tipo, y Selander (1960a) establece el género Paniculolytta, en el que únicamente integra a $L$. sanguineoguttata, que a su vez designa como especie-tipo. De la lectura comparada de las descripciones de estos taxones, se concluye que los dos autores están describiendo el mismo género y al poco tiempo, el propio Kaszab (1963: 335) incluye a Paniculolytta en la sinonimia de Denierota, que ostenta la prioridad nomenclatural. En ninguno de sus trabajos Kaszab $(1959,1963,1969)$ hace referencia alguna a $L$. sanguineoguttata, ni tampoco Selander (1960a) menciona a $L$. kraatzi en el suyo, ni en la revisión de Lytta que publica simultáneamente (Selander, 1960b), donde sí alude a Paniculolytta sanguineoguttata. Parecería pues evidente que ninguno de los autores mencionados hubiese examinado ejemplares de las dos especies al mismo tiempo, ya que en caso contrario las hubieran incluido en sus respectivos géneros, cosa que no hicieron. Sin embargo, posteriormente Selander (1991: 69), sin precisar si estudió material tipo de L. kraatzi, consideró sinónimas ambas especies, aunque sin formalizar la sinonimia ni realizar comentario alguno que avalase tal criterio.

En la descripción original de $L$. sanguineoguttata, Haag-Rutenberg (1880: 40) indica que el material que sirvió para la definición de la especie procedía de "Guatemala" (localidad-tipo). Champion (1892) redescribe detalladamente la especie a partir de material guatemalteco, detallando sus caracteres peculiares que posteriormente sirvieron para la caracterización de Denierota (como por ejemplo presentar un pronoto muy alargado, campaniforme, los trocánteres del macho con un mechón de sedas rígi- 

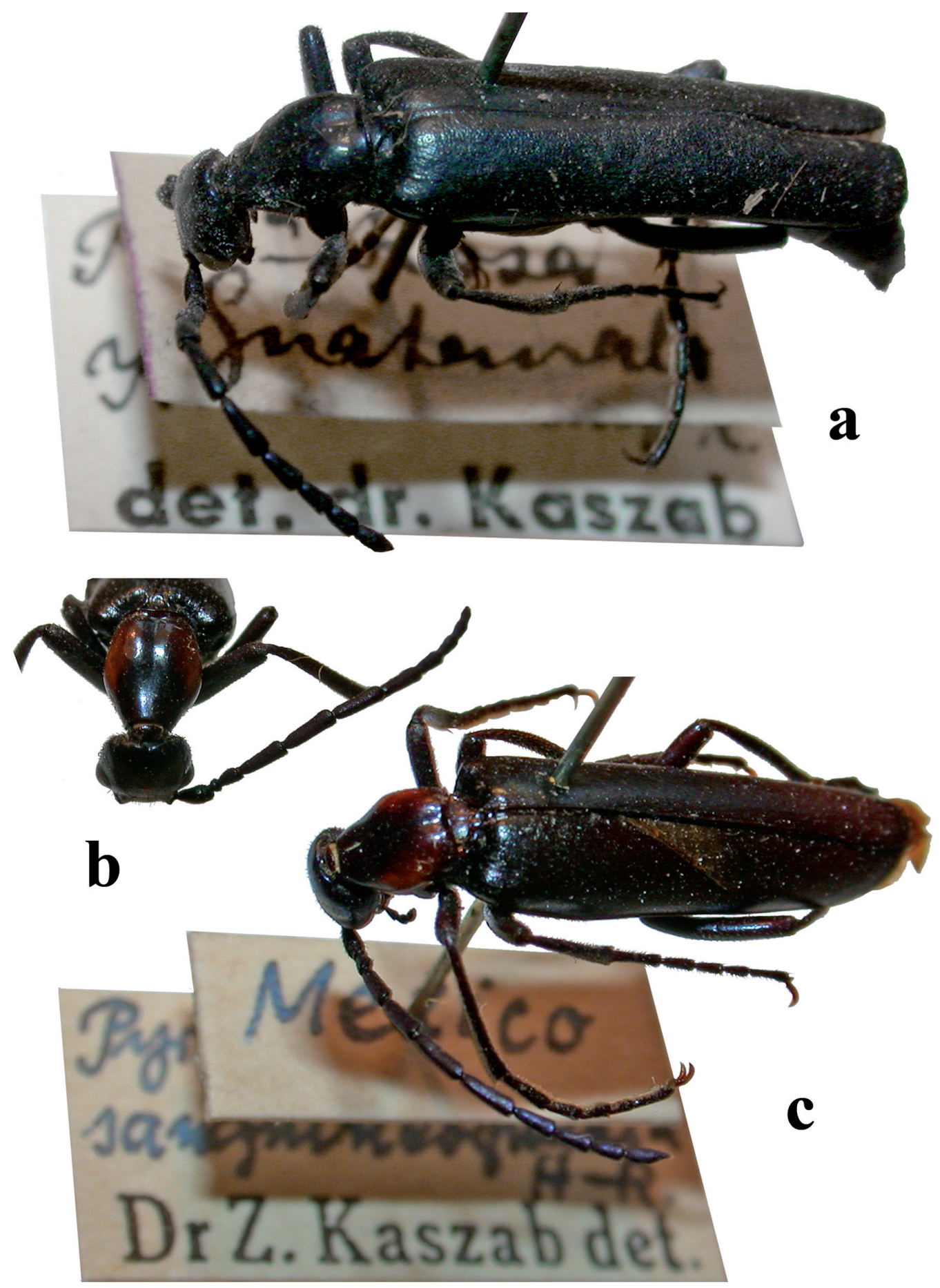

Fig. 1.- Hábitus de Denierota kraatzi (Haag-Rutenberg, 1880): a) Guatemala (Col. HNHM), ejemplar identificado por Z. Kaszab como "Pyrota kraatzi H-R"; b y c) México (Col. HNHM), ejemplar identificado por Z. Kaszab como "Pyrota kraatzi ab. sanguineoguttata H-R".

Fig. 1.- Habitus of Denierota kraatzi (Haag-Rutenberg, 1880): a) Guatemala (Col. HNHM), specimen identified by Z. Kaszab as "Pyrota kraatzi H-R"; b and c) México (Col. HNHM), specimen identified by Z. Kaszab as "Pyrota kraatzi ab. sanguineoguttata H-R". 
das -que Champion considera espinas-, el primer segmento de los tarsos medios del macho con un tubérculo grueso que se extiende ventralmente formando un ángulo recto con el tarso y el pigidio del macho con un proceso medio dilatado apicalmente), e indica su variabilidad en la coloración, que incluye tanto individuos completamente verde-azulados, como otros de coloración más mate con manchas rojizas en los laterales del pronoto. Selander (1960a), años después, redescribe la especie a partir de material guatemalteco, designa lectotipo en la colección Haag-Rutenberg del Zoologische Staatsammlung de Münich, e indica que los ejemplares pueden presentar o no las manchas rojizas. Por otra parte, Pic (1916) describe Lytta (Pyrota) submetallica de Guatemala y la diferencia de las demás especies de Pyrota Dejean, 1834 por su coloración verde-metálica y la forma alargada del cuerpo con el pigidio prominente. Denier (1934), en su revisión del género Pyrota, indica que la especie de Pic se adscribe al género Lytta, siendo afín a $L$. sanguineoguttata y descarta su inclusión en el género Pyrota. Ya en su catálogo de los Meloidae de América, Denier (1935a) incluye formalmente a L. submetallica en la sinonimia de $L$. sanguineoguttata, donde se mantiene hasta la fecha. Pic (1916: 10) por error desliza el nombre de $L$. sanguineovittata en lugar de L. sanguineoguttata.

En la descripción de L. kraatzi, Haag-Rutenberg (1880: 22) indica que se desconoce la procedencia del material que utilizó para la descripción de la especie ("Patria ignota"), aunque supone que podría provenir del Brasil, tal vez como consecuencia de la comparación que hace en ese mismo párrafo (HaagRutenberg, 1880: 23) con Lytta herculeana Germar, 1824 [actualmente Brasiliota herculeana (Germar, 1824), único representante y especie-tipo, por designación original, del género Brasiliota Kaszab, 1959, restringido al centro y sur de Brasil (Pinto \& Bologna, 1999)]. Beauregard (1890: 494, nota 2) recoge este último comentario de Haag-Rutenberg (1880), e indica además que podría tratarse de una especie del género Pyrota atendiendo a la descripción original, mientras que Champion (1892) no hace referencia alguna a esta especie en su obra.

Recientemente hemos tenido la oportunidad de revisar el material de Denierota conservado en la colección del HNHM de Budapest que, sin duda, fue el utilizado en la descripción del género. Este material incluye tres ejemplares. Uno de ellos, procedente de Santa Rosa (Guatemala) y otro de México (sin más precisión) portan etiquetas de identificación de Z. Kaszab como "Pyrota kraatzi Haag”, ambos poseen el pronoto y los élitros de coloración uniforme, azulado verdoso (Fig. 1a). El tercer ejemplar, también de México (sin más precisión), porta una etiqueta de identificación de $\mathrm{Z}$. Kaszab como "Pyrota kraatzi ab. sanguineoguttata Haag" y presenta una coloración pardonegruzca con los lados del pronoto y propleuras de color rojizo (Fig. 1b, c). La comparación de estos ejemplares entre sí y a su vez con la descripción de Paniculolytta sanguineoguttata proporcionada por Selander (1960a), no deja lugar a dudas sobre la identidad taxonómica de los ejemplares estudiados tanto por Kaszab (1959) como por Selander (1960a), concretándose la única diferencia diagnóstica entre ellos en la ausencia o presencia (con extensión variable y límites difusos) de manchas rojizas en los laterales del pronoto y en la coloración general. Por lo tanto, con independencia del nombre válido aplicable, el taxón específico sobre el que se describieron los géneros Denierota y Paniculolytta es el mismo (como ya indicó Selander, 1991).

Además, la descripción de Champion (1892) y las identificaciones de Z. Kaszab en los ejemplares del HNHM asignan inequívocamente las dos formas de coloración, azul-verde submetálico y pardonegruzca con manchas rojizas a un único taxon, Cantharis sanguineoguttata en el caso de Champion, Denierota kraatzi en el caso de Kaszab, autor este último que según la etiqueta de determinación examinada (ejemplar mexicano), subordina $L$. sanguineoguttata a una simple variedad de $D$. kraatzi. Por otra parte, en la colección MAB existen dos ejemplares de Denierota, indiferenciables entre sí, excepto por la coloración, ya que cada uno corresponde a un morfotipo diferente, capturados en la misma localidad: Quisache (Municipio Acatenango, Guatemala). En ausencia de otros rasgos diagnósticos, la existencia de simpatría estricta cuestiona el reconocimiento de dos especies distintas para estas variantes de coloración.

El problema surge por lo tanto a la hora de aplicar el nombre adecuado a estos ejemplares. La descripción original de L. sanguineoguttata realizada a partir de ejemplares procedentes de Guatemala (Haag-Rutenberg, 1880) se ajusta claramente a la de los ejemplares de Guatemala y México con tonos rojizos estudiados por nosotros en la colección del HNHM; la descripción e ilustración presentada por Champion (1892) permite asignar inequívocamente los ejemplares del HNHM a su concepto de Cantharis sanguineoguttata. Igualmente estos ejemplares encajan con la descripción de Selander (1960a) de Paniculolytta sanguineoguttata. Por otra parte, los ejemplares de coloración verdosa, más metálicos, como el ejemplar estudia- 
do por nosotros de Chiapas, o el que usó Kaszab en la diagnosis de Denierota del HNHM, corresponderían a la descripción riginal de $L$. kraatzi.

Ni Kaszab (1959) ni Selander (1960a) indican que hayan revisado el tipo de L. kraatzi. Es muy probable que Kaszab haya considerado, tras la lectura de las descripciones de Haag-Rutenberg (1880), que los dos nombres correspondían a un único taxon. Puesto que L. kaatzi se describe unas páginas antes, Kaszab habría usado ese nombre por considerarlo prioritario. Mientras que Selander, que sí revisó el material de L. sanguineoguttata de Haag-Rutenberg designando lectotipo, habría seguido la nomenclatura de Champion (1892).

A raíz de una consulta para la localización de los ejemplares tipo de Haag-Rutemberg (gracias a la cortesía de M. A. Bologna) hemos tenido la oportunidad de revisar fotografías enviadas por Michael Balke de los respectivos tipos de L. sanguineoguttata y L. kraatzi del Zoologische Staatssammlung de Münich (Alemania). Los tipos representan las dos variantes de coloración de Denierota, existentes en las colecciones del HNHM y de MAB, y los rasgos que es posible examinar coinciden totalmente entre sí y con los de los ejemplares estudiados. Por lo tanto concluimos que los tipos de $L$. sanguineoguttata y $L$. kraatzi corresponden a un único taxon, y por lo tanto ambos nombres son sinónimos. Es muy posible que Selander (1991) ya hubiese llegado a esta conclusión cuando designó lectotipo de L. sanguineoguttata y por ello indicó que ambos nombres corresponderían a una única especie.

Ante esta situación, establecemos formalmente la sinonimia de L. sanguineoguttata con L. kraatzi, ya que ambos nombres están disponibles y fueron publicados por Haag-Rutenberg (1880) en el mismo trabajo. Con esta actuación, al mantener Lytta kraatzi la prioridad, no se requiere el cambio de especie-tipo del género Denierota.

Por lo tanto, el género pasa a ser monotípico y endémico de México, Guatemala y El Salvador, aunque John D. Pinto (com. pers., 2008) indica que existe una especie aún sin describir en México. La sinonimia de D. kraatzi quedaría por lo tanto como sigue:

Denierota kraatzi (Haag-Rutenberg, 1880)

Lytta kraatzi Haag-Rutenberg, 1880: 22

Lytta sanguineoguttata Haag-Rutenberg, 1880: 40

Cantharis kraatzi (Haag-Rutenberg, 1880): Beauregard, 1890: 494

Cantharis sanguineoguttata (Haag-Rutenberg, 1880): Beauregard, 1890: 496

Lytta (Pyrota) submetallica Pic, 1916: 10

Lytta sanguineovittata Pic, 1916: 10 (error pro L. sanguineoguttata)
Epicauta kraatzi (Haag-Rutenberg, 1880): Borchmann, 1917: 76

Denierota kraatzi (Haag-Rutenberg, 1880): Kaszab, 1959: 106

Paniculolytta sanguineoguttata (Haag-Rutenberg, 1880): Selander, 1960: 81

Denierota sanguineoguttata (Haag-Rutenberg, 1880): Selander, 1991: 69

Pyrota decorata (Haag-Rutenberg, 1880)

MéXico: Chiapas: “Zapotal 1850'. 3 mi. S Tuxtla Gut., 5-X53, arid low tropics (R.B. Selander leg.) (Pyrota decorata Haag, R.B. Selander det.) [HNHM]. Chis. 2 ejemplares (RF) (136) (Dampf) [MAB].

Especie distribuida desde el sur de México hasta Panamá (Champion, 1892; Borchmann, 1917; Maes \& Huether, 2007). En México se ha citado en los estados de Oaxaca y Veracruz (Champion, 1892; Denier, 1934), por lo que este registro de Zapotal es el primero para el Estado de Chiapas. Los dos ejemplares de la colección MAB sólo indican el Estado sin más precisión.

\section{Cissites auriculata (Champion, 1892)}

MéXICo: ChIAPAS: Municipio La Trinitaria: $30 \mathrm{~km}$ al SE de La Trinitaria: 29-IX-1994, 1 ejemplar (R. Jones leg.) [ECOSUR]. Municipio Ocozocuautla: Selva El Ocote: Río Encajonado: 8/15VI-1994, 1 ejemplar (B. Gómez leg.) [ECOSUR]. Municipio Tapachula: El Espolón: 15-II-1974, 1 ejemplar (A.R.R. leg.) [ECOSUR].

Especie distribuida desde Texas (EUA) y México hasta Costa Rica, con citas en Guatemala y Nicaragua (Enns, 1958; Maes, 1989; Lewis, 2004). En México se ha citado en Colima, Guerrero, Jalisco, Morelos, Nayarit, Oaxaca, Puebla, Sinaloa y Veracruz (ver García-París et al., 2007), sin que existiese ningún registro previo para Chiapas.

\section{Tetraonyx frontalis Chevrolat, 1833}

MÉXICo: ChIAPAS: Huixtla: Colonia Morelos: V-1976, 1 ejemplar [ECOSUR].

$\mathrm{Su}$ distribución geográfica incluye México, Guatemala, El Salvador y el sur de los Estados Unidos (Selander, 1983a). En México se ha citado previamente en el Distrito Federal, Guerrero, Oaxaca, Puebla y Veracruz (Chevrolat, 1833; Dugès, 1869a; Champion, 1892; Vaurie, 1950; Selander, 1983a). La localidad señalada constituye el primer registro para Chiapas. 


\section{Comentarios adicionales}

Además de las especies señaladas, en Chiapas se ha registrado la presencia de las siguientes especies (véase García-París et al., 2007): Epicauta (Epicauta) basimacula (Haag-Rutenberg, 1880), Epicauta (Epicauta) carmelita (Haag-Rutenberg, 1880), Epicauta (Epicauta) mixta Dugès, 1889, Epicauta (Epicauta) unilineata Champion, 1892, Epicauta (Macrobasis) evanescens Champion, 1892, Lytta (Adicolytta) eucera (Chevrolat, 1834), Meloe (Meloe) nebulosus Pinto et Selander, 1970, Pyrota divirgata (Villada et Peñafiel, 1867), Pyrota rugulipennis Champion, 1892, Pyrota tenuicostatis (Dugès, 1877), Hornia mexicana Dugès, 1886 y Nemognatha chrysomeloides (Linnaeus, 1763), con lo que la cifra de especies citadas en el Estado asciende, hasta el momento, a un total de 32.

Esta cifra apenas constituye el $12.5 \%$ de las 258 especies de Meloidae presentes en México (una vez añadidas las dos especies citadas por primera vez en este trabajo) (García-París et al., 2007), aunque representa algo más del $70 \%$ de las especies conocidas en Guatemala (Champion, 1892). Tal como ocurre en otras zonas del Planeta (Bologna, 1991), la mayor diversidad de Meloidae en América del Norte se centra en las zonas áridas y semiáridas, motivo por el cual es esperable que la diversidad relativa de Meloidae sea muy baja en Chiapas y Guatemala, donde faltan las zonas áridas amplias. Por otra parte el género Lytta, muy diverso en zonas del centro y norte del país, sólo está representado al sur del istmo de Tehuantepec por una especie de amplia distribución, L. eucera, que desde el norte de México alcanza el extremo septentrional de América del Sur (Selander, 1960b; Pinto \& Bologna, 1999; García-París et al., 2007) por lo que no es esperable que se incremente el número de citas de este género.

A estas peculiaridades biológicas de la familia Meloidae, hay que añadir sin duda el déficit debido a la falta de prospecciones adecuadas. Las mayores diferencias en la composición de la fauna de Chiapas con respecto a la de Guatemala residen en la casi total ausencia de registros de la tribu Nemognathini en Chiapas. Esta ausencia se debe sin duda a defectos de muestreo, ya que muchas de las especies de Nemognathini presentes en Guatemala también lo están en México, especialmente en Oaxaca (ejemplo Zonitis oaxacae Enns, 1959). Sin embargo, ninguna de estas especies se han citado hasta el momento en el Estado de Chiapas, que únicamente cuenta con dos representantes registrados de esta tribu: Hornia mexicana Dugès, 1886 y Nemognatha chrysomeloides (Linnaeus, 1763).
Ninguna de las especies de la familia Meloidae encontradas en Chiapas es endémica del Estado, y tan sólo la subespecie Hornia mexicana blomi MacSwain, 1958 se encuentra exclusivamente en el mismo (posiblemente como consecuencia de la dificultad de muestreo del género Hornia, cuyos ejemplares siempre se localizan dentro o junto a los nidos de los Apoidea hospedadores, MacSwain, 1958).

En cuanto a la fenología, de acuerdo con los datos disponibles, la actividad de los adultos de la familia Meloidae parece concentrarse en dos periodos, uno entre finales de abril y mediados de julio, y otro desde finales de septiembre a primeros de noviembre, con registros aislados en febrero $(C$. auriculata), en agosto (D. sanguineoguttata) y en diciembre (M. tropicus).

\section{AGRADECIMIENTOS}

Nuestro agradecimiento a Antonio Muñoz (ECOSUR, San Cristóbal de las Casas) por las facilidades prestadas para la revisión de la Colección Entomológica del ECOSUR; a Elizabeth Mejorada (Instituto de Bilología, DF) por su amabilidad y apoyo durante la revisión de la colección CNIN-IBUNAM; a Marco A. Bologna (Università Roma Tre) por ayudarnos a resolver el problema de la localización de los tipos de Denierota y permitirnos estudiar su imprescindible colección de Meloidae; a Otto Merkl (HNHM, Budapest) por las facilidades prestadas para el examen de material de la colección del HNHM; a John D. Pinto (University of California, Riverside) por su insistencia para la resolución del problema de Denierota; a Michael Balke (Zoologische Staatssammlung, Münich) por su amabilidad al proporcionarnos fotos digitales de los tipos de L. kraatzi y L. sanguineoguttata y finalmente a Pepe Fernández (MNCN, Madrid) por su permanente ayuda en la edición del manuscrito y en la solución de problemas bibliográficos.

Este trabajo ha contado para su realización con el proyecto CGL2007-64621 del Ministerio de Educación y Ciencia (España), lo que nos ha facilitado el apoyo logístico necesario. La visita de MGP a la colección del Magyar Természettudományi Múzeum (HNHM) en Budapest ha sido financiada por el proyecto Synthesys «European Commission's Research Infrastructure Action». La visita de MGP a la colección del Instituto de Biología de la UNAM y al ECOSUR de San Cristóbal de las Casas ha sido posible gracias a la "Estancia Libre" concedida a MGP en el marco del convenio CSICUNAM (2007).

\section{Referencias}

Adams, C. L. \& Selander, R. B., 1979. The biology of blister beetles of the Vittata group of the genus Epicauta (Coleoptera, Meloidae). Bulletin of the American Museum of Natural History, 162: 139-266.

BAtes, H. W., 1884. Families Carabidae, Cicindelidae (Suppl.). In: F. E. Godman \& O. Salvin (eds.). Biologia Centrali-Americana. Coleoptera, Vol. 1, pt. 1. London: 256-299. 
Beauregard, H., 1890. Les insectes vésicants. Félix Alcan. Paris. Xvi + 544 pp., 34 láms.

BlackWELDER, R. E., 1945. Checklist of the coleopterous insects of Mexico, Central America, the West Indies, and South America. Part 3. United States National Museum Bulletin, 185: 343-550.

Bologna, M. A., 1991. Fauna de Italia. Coleoptera Meloidae. Calderini. Bologna. xiv + 541 pp.

Borchmann, F., 1917. Pars 69. Meloidae, Cephaloidae. In: E. Schenkling (ed.). Coleopterorum Catalogus auspiciis et auxilio W. Junk. Junk. Berlin. 208 pp.

Champion, G. C., 1891. Families Mordellidae, Rhipidophoridae, Meloidae. In: F. E. Godman \& O. Salvin (eds.). Biologia Centrali-Americana. Coleoptera Hete-romera. Vol. 4, pt. 2. London: 257-368.

Champion, G. C., 1892. Family Meloidae. In: F. E. Godman \& O. Salvin (eds.). Biologia CentraliAmericana. Coleoptera Heteromera. Vol. 4, pt. 2. London: 369-448, láms. 17-21.

Chevrolat, L. A. A., 1833. Coléoptères du Mexique. 1er. Fascicule. G. Silbermann. Strasbourg. Sin paginar.

Chevrolat, L. A. A., 1834. Coléoptères du Mexique.

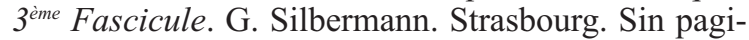
nar.

CINZ (Comisión Internacional de Nomenclatura Zoológica). 2000. Código Internacional de Nomenclatura Zoológica, $4^{a}$ edición. Museo Nacional de Ciencias Naturales (CSIC) e International Trust for Zoological Nomenclature. Madrid. 156 pp. [Traducción al español de: ICZN. 2000. International Code of Zoological Nomenclature. 4th ed. International Trust for Zoological Nomenclature. London. 306 pp.]

Denier, P., 1934. Contribución al estudio de los meloidos americanos. II. Ensayo de clasificación de la Pyrota (Dej.) Lec. basada en los caracteres sexuales secundarios de los machos. Revista de la Sociedad Entomológica Argentina, 6: 49-75.

DENIER, P., 1935a. Coleopterorum americanorum familiae meloidarum. Enumeratio synonymica. Revista de la Sociedad Entomológica Argentina, 7: 139-176.

DENIER, P., 1935b. Estudios sobre meloidos americanos. Apuntes críticos de sistemática y de nomenclatura. Revista Argentina de Entomología, 1: 15-28.

DuGÈs, E., 1869a. Descripción de algunos meloideos indígenas. La Naturaleza, 1: 100-113. [entrega 4 pág. 100; entrega $5^{\mathrm{a}}$ pág. 105].

DugÈs, E., 1869b. Descripción de algunos meloideos indígenas. La Naturaleza, 1: 125-128. [entrega $6^{\text {a }}$ pág. 125: 1869]

DugÈs, E., 1870. Descripción de algunos meloideos indígenas. La Naturaleza, 1: 161-171, láms. 1-2. [entrega $8^{a}$ pág. 161: 1870]

DugÈs, E., 1877. Descripción de algunos meloideos indígenas. La Naturaleza, 4: 57-67, lám. 2.
DuGÈs, E., 1886. Note pour servir a la classification des méloïdes du Mexique. Bulletin de la Societé Zoologique de France, 11: 578-582.

DugÈs, E., 1889. Sinopsis de los meloideos de la República Mexicana. Anales del Museo Michoacano, 2: 49-113 + 107bis-114bis + 194-195.

ENNS, W. R., 1958. Distribution records of two American species of Cissites (Coleoptera-Meloidae). The Coleopterists'Bulletin, 12: 61-64.

EnNs, W. R., 1959. Two new species of blister beetles with an additional new designation (Meloidae). The Coleopterists'Bulletin, 13: 13-17.

García-París, M., Buckley, D. \& Parra-Olea, G., 2007. Catálogo taxonómico-geográfico de los coleópteros de la familia Meloidae de México. Graellsia, 63(2): 165-258.

HaAg-Rutenberg, J. G., 1880. Beiträge zur Kentniss der Canthariden. Deutsche Entomologische Zeitschrift, 24: 17-90.

HorN, G. H., 1885. Studies among the Meloidae. Transac-tions of the American Entomological Society, 12: 107-116, lám. 5

KaszaB, Z., 1959. Phylogenetische Beziehungen des Flügelgeäders der Meloiden (Coleoptera), nebst Beschreibung neuer Gattungen und Arten. Acta Zoologica Academiae Scientiarum Hungaricae, 5: 67-114.

KaszaB, Z., 1963. Studien über Meloiden (Coleoptera). Annales Historico-Naturales Musei Nationalis Hungarici, 55: 335-346.

KaszaB, Z., 1969. The system of the Meloidae (Coleoptera). Memorie della Società Entomologica Italiana, 48: 241-248.

LEwIS, E., 2004. A United States record for the genus Cissites Latreille (Coleoptera: Meloidae: Zonitini). The Coleopterists'Bulletin, 58(4): 635-636.

Linsley, E. G., MacSwain, J. W. \& Michener, C. D., 1980. Nesting biology and associates of Melitoma (Hyme-noptera, Anthophoridae). University of California Publications in Entomology, 90: 1-45.

MacSwain, J. W., 1958. Taxonomic and biological observations on the genus Hornia (Coleoptera: Meloidae). Annals of the Entomological Society of America, 51: 390-396.

Maes, J. M., 1989. Catálogo de los insectos controladores biológicos en Nicaragua. VII. Insectos depredadores del orden Coleoptera. Super Familia Meloidea. Revista Nicaragüense de Entomología, 9: 111-120.

Maes, J. M. \& Huether, J. P., 2007. Catálogo ilustrado de los Meloidae (Coleoptera) de Nicaragua y otras especies contenidas en el Museo Entomológico de León. Revista Nicaragüense de Entomología, 67(Suple-mento 3): 1-90.

Mathieu, J. M., 1983. Two new Epicauta from Mexico (Coleoptera: Meloidae). Proceedings of the Entomological Society of Washington, 85: 156-160. 
PIC, M., 1916. Descriptions abregées diverses. Mélanges Exotico-Entomologiques, 21: 2-20.

Pic, M., 1924. Nouveautés diverses. Mélanges ExoticoEntomologiques, 42: 1-32.

PinTo, J. D., 1984. Cladistic and phenetic estimates of relationship among genera of eupomphine blister beetles (Coleoptera: Meloidae). Systematic Entomology, 9: $165-182$.

Pinto, J. D., 1991. The taxonomy of North American Epicauta (Coleoptera: Meloidae), with a revision of the nominate subgenus and a survey of courtship behavior. University of California Publications in Entomology, 110: i-x, 1-372, 40 láms.

Pinto, J. D. \& Bologna, M. A., 1999. The New World genera of Meloidae (Coleoptera): a key and synopsis. Journal of Natural History, 33: 569-620.

Pinto, J. D. \& Selander, R. B., 1970. The bionomics of blister beetles of the genus Meloe and a classification of the New World species. Illinois Biological Monographs, 42: 1-222.

SÁnchez Soto, S., 2001. Ocurrencia de Lamprosema indicata (Fabricius) (Lepidoptera: Pyralidae) en el cultivo de frijol en Tabasco, México. Folia Entomológica Mexicana, 40(2): 281-282.

SElAnDER, R. B., 1960a. Restriction of the genus Lytta Fabricius (Meloidae). The Coleopterists'Bulletin, 14: 80-86.

Selander, R. B., 1960b. Bionomics, systematics and phylogeny of Lytta, a genus of blister beetles (Coleop-tera: Meloidae). Illinois Biological Monographs, 28: i-iv, 1-295.

SElander, R. B., 1983. An annotated catalog of blister beetles of the tribe Tetraonycini (Coleoptera, Meloidae). Transactions of the American Entomological Society, 109: 277-293.

SELANDER, R. B., 1991. On the Nomenclature and Classification of the Meloidae (Coleoptera). Insecta Mundi, 5(2): 65-94.
Selander, R. B. \& Agafitei, N. J., 1982. First-instar larva of Epicauta funesta (Chevrolat) (Coleoptera: Meloidae). Coleopterists'Bulletin, 36(2): 200-2005.

VAurie, P., 1950. The blister beetles of North Central Mexico (Coleoptera: Meloidae). American Museum Novitates, 1477: 1-68.

Werner, F. G., 1949. Epicauta diversicornis and its allies in the Neotropical Region (Coleop., Meloidae). Psyche, 56(2): 74-80.

Werner, F. G., 1973. Three new Epicauta from Mexico (Coleoptera: Meloidae). Proceedings of the Entomolo-gical Society of Washington, 75: 458-463.
Recibido, 28-X-2008

Aceptado, 13-V-2009

Publicado, 29-VI-2009 